\title{
A computational approach to the design of a cryogenic turbine blade profile
}

\author{
Subrata Kr. Ghosh ${ }^{1 *}$, R. K. Sahoo ${ }^{2}$, Sunil K. Sarangi ${ }^{2}$ \\ ${ }^{1 *}$ Indian School of Mines University, Dhanbad Jharkhand, India, \\ ${ }^{2}$ National Institute of Technology, Rourkela, Orissa, India \\ "Corresponding author (e-mail: subratarec@yahoo.co.in)
}

\begin{abstract}
The present day cryogenic gas turbines are in high use as they meet the growing needs for low pressure cycles. This calls for improved methods of blade profile design. The present study is aimed at the design of the blade profile of mixed flow impellers with radial entry and axial discharge. In this paper, details of a computer code have been developed for designing such blade profile. The computational procedure developed describes the three-dimensional contours of the blades for the turbine wheel. The flow of fluid in a turbine blade passage depends on the length of the flow path and the curvature of such path. This has been given due weightage. Effect of some of the turbine operating and design parameters on the flow path and its curvature have been analyzed and presented. Optimum solution for some of the free parameters and angles between velocity components was found. On that basis, co-ordinates of this blade profile were computed. Although manufacturers design the blade profiles for their production, till date no academic literature is available for this purpose. The work may help researchers for further developmental work on this topic as well as the manufacturers of cryogenic expanders.
\end{abstract}

Keywords: Radial turbine, Blade profile parameter, Meridional streamlength, Axial coordinate, Radial coordinate, Angular coordinate

\section{Introduction}

A medium or large cryogenic system needs many components - compressors, heat exchanger, expansion turbine, instrumentation, vacuum vessel, etc. At present, in many developing countries, most of the cryogenic system components are sourced from industrialized nations in view of their high demand locally. This has enormous foreign exchange implications; these spending could be preserved for local industrial growth. Thus, there is a need for countries to be self reliant in this vital technology. In India, all components of cryogenic systems are available locally; however the whole plant is being imported due to lack of the single component, expansion turbine. Successful development of a turboexpander strongly depends on the performance of the turbine wheel. The complete design of turbomachinery rotors requires aerodynamic analysis of the flow path and structural analysis of the rotor including the blades and hub (Bruce, 1998). In order to develop such a design system, the rotor geometry generation procedure should be clearly understood. A computerized method for specifying the shape of impellers and guide vanes has been described by different literature (Wallace et al., 1975; Whitfield, 1978; Casey, 1983; Strinning, 1995). It is necessary that all machine components operate efficiently. This can be achieved by selecting the most suitable combination of design parameters with the following conditions ensured - the highest possible value of efficiency, uniform and steady operating conditions and an easy manufacture of the blades. For the turbine blade design $K_{e}$ varies from 0.75 to 1 and $K_{h}$ varies from 1 to 20 (Balje, 1970). Turbine blade surface deterioration is strongly dependent on the turbine geometry and blade surface material. A numerical model has been developed to show the effects of aerodynamic forces in the three dimensional turbine flow field (Hamed et al., 2005).

The paper is devoted to the design of the blade profile of mixed flow impellers with radial entry and axial discharge. The computational process aims at defining a blade profile that maximises the performance with highest possible value of efficiency, uniform and steady operating conditions and easy manufacture of the blades. The detailed procedure describes computation of the 
three-dimensional contours of the blades and simultaneously determines the velocity, pressure and temperature profiles in the turbine wheel. The magnitude of the velocity and change in its direction determine the optimum blade profile of the turbine. In a word, in this paper a parametric study has been carried out to determine the optimum blade profile for given specifications.

\section{Basic design of turbine wheel}

The design of turbine wheel has been done following the method outlined by some authors (Balje, 1981; Kun and Sentz, 1985), which are based on the well known "similarity principles". The similarity laws state that for any given Reynolds number, Mach number and Specific heat ratio of the working fluid to achieve optimized geometry for maximum efficiency, two dimensionless parameters: specific speed and specific diameter uniquely determine the major dimensions of the wheel and its inlet and exit velocity triangles. Specific speed $\left(n_{s}\right)$ and specific diameter $\left(d_{s}\right)$ are defined as:

$$
\begin{array}{ll}
\text { Specific speed } & \mathrm{n}_{\mathrm{s}}=\frac{\omega \times \sqrt{\mathrm{Q}_{3}}}{\left(\Delta \mathrm{h}_{\mathrm{in}-3 \mathrm{~s}}\right)^{3 / 4}} \\
\text { Specific diameter } & \mathrm{d}_{\mathrm{s}}=\frac{\mathrm{D}_{2} \times\left(\Delta \mathrm{h}_{\mathrm{in}-3 \mathrm{~s}}\right)^{1 / 4}}{\sqrt{\mathrm{Q}_{3}}}
\end{array}
$$

The basic input parameters for the cryogenic expansion turbine system are:

$\begin{array}{ll}\text { Working fluid: } & \text { Air } \\ \text { Discharge pressure: } & 1.5 \mathrm{bar} \\ \text { Turbine inlet temperature: } & 122 \mathrm{~K} \\ \text { Throughput: } & 67.5 \mathrm{~nm}^{3} / \mathrm{hr} \\ \text { Turbine inlet pressure: } & 6.0 \mathrm{bar} \\ \text { Expected efficiency: } & 75 \%\end{array}$

From Balje (1981), the peak efficiency of a radial inflow turbine corresponds to the values of:

$$
n_{s}=0.54 \text { and } d_{s}=3.4
$$

Substituting these values in equations (1) and (2) respectively, yields

$$
\begin{array}{ll}
\text { Rotational speed } & \omega=22910 \mathrm{rad} / \mathrm{s} \\
\text { Wheel diameter } & D_{2}=16.0 \mathrm{~mm} \\
\text { Power produced } & \mathrm{P}=0.9 \mathrm{KW}
\end{array}
$$

The ratio of exit tip diameter to inlet diameter should be limited to a maximum value of 0.70 (Dixon, 1978; Rohlik and Harold, 1968) to avoid excessive shroud curvature. Corresponding to the peak efficiency point (Balje, 1981):

$$
\xi=\mathrm{D}_{\text {tip }} / \mathrm{D}_{2}=0.676 \quad D_{\text {tip }}=10.8 \mathrm{~mm}
$$

In order to determine the optimum turbine geometry, various studies have been conducted and published in relation to the rotor blade profile (Thakker and Abdulhadi, 2007). According to Rohlik and Harold (1968), the exit hub to tip diameter ratio should maintained above a value of 0.4 to avoid excessive hub blade blockage and energy loss. Kun and Sentz (1985) have taken a hub ratio of 0.35 citing mechanical considerations.

$$
\lambda=D_{\text {hub }} / D_{\text {tip }}=.425 \quad D_{h u b}=4.6 \mathrm{~mm}
$$

Recommendation has given on the choice of number of blades by ensuring that the flow does not stagnate the pressure surface (Denton, 1996). For small turbines, the hub circumference at exit and diameter of milling cutters available determine the number of blades. In this design the number of blades $\left(Z_{t r}\right)$ are chosen to be 10 , and the thickness of the blades to be 0.6 mm throughout.

\section{Determination of meridional geometry}

In selecting the most suitable combination of parameters, the rotor contour (tip and hub streamline) and the change of the flow angle with flow path length are determined when a pressure balanced flow path is desired. This also means that an arbitrary 
selection of the rotor contour and angular change with flow path length is likely to yield a design with potentially high transverse pressure gradients.

Thus complete pressure balance in practical flow path designs will be nearly impossible. The three dimensional effects can, however, be minimized by keeping the relative velocity gradient low, i.e., by providing a high blade number in that portion of the flow path where the suction side and pressure side streamlines begin to diverge, up to the point where the flow path inclination angle $\delta$ approaches $90^{\circ}$.

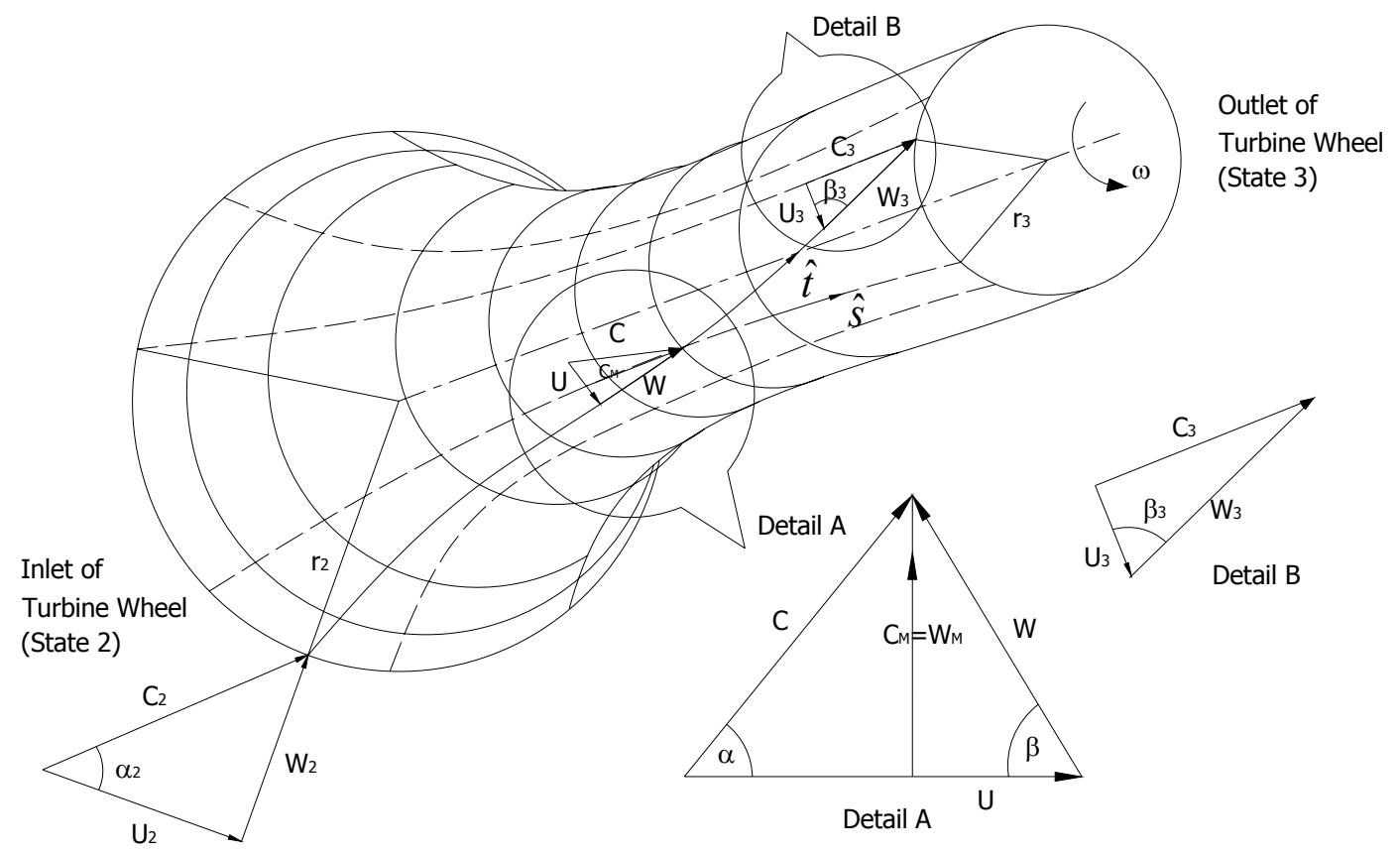

Figure 1. Illustration of flows in radial axial impeller

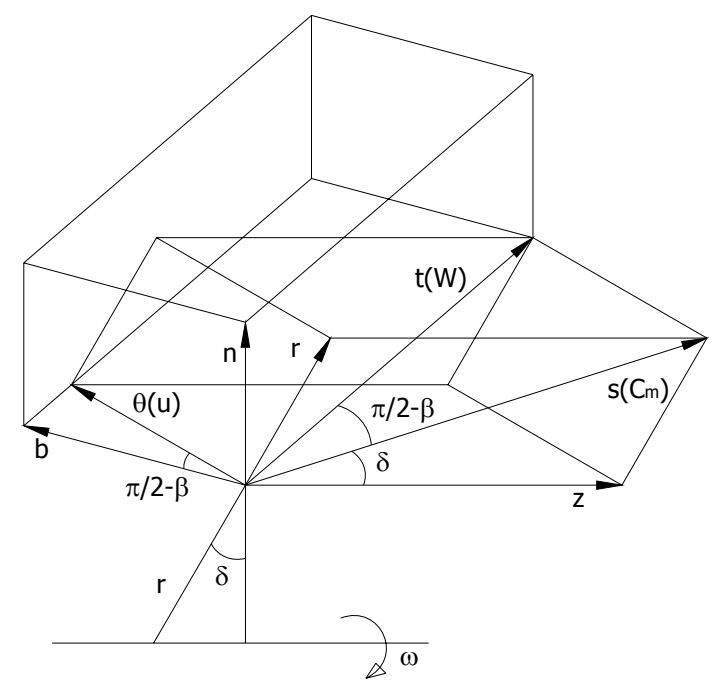

Figure 2. Coordinate system

The concave and convex profiles between blades are designed to reduce the pressure difference between the pressure and suction surfaces near the endwall, as this causes the secondary flow and the end wall boundary layer to conform as much as possible to the mainstream flow direction. At the same time, the trailing edge profile is designed such that as much of the primary flow as possible leaves the blade smoothly in the mainstream flow direction (Eisaku et al., 2009).

The design of blade profile is usually carried out by Hasselgruber's approach (Hasselgruber, 1958). The equations are derived in a body fitted orthogonal coordinate system $(t, b, n)$. The coordinate $t$ is the direction along the central streamline, the coordinate $b$ is the lateral coordinate between the suction and the pressure surfaces and the coordinate $\mathrm{n}$ refers to depth of the flow path in the turbine passage. The author also defines the meridional coordinate $s$ to correlate the body fitted coordinate system with the 
cylindrical coordinate system $(\mathrm{r}, \theta, \mathrm{z})$ where the s coordinate lies on the r-z plane. Figure 1 and Figure 2 show the flow velocity and coordinate transformation respectively.

\subsection{Assumptions}

The blade profiles have been worked out using the technique of Hasselgruber (1958), which was also employed by others (Kun and Sentz, 1958; Balje, 1993, 1981). Hasselgruber's approach of computation for the central streamline is based on some key assumptions such as:

(i) Constant acceleration of the relative velocity

The blades of pure radial impellers are so shaped that it always gives a constant retardation to the relative velocity. The acceleration of relative velocity from wheel inlet to exit follows a power law relation. The substantial derivative under steady state condition results,

$$
\frac{D W}{D \tau}=W \frac{\partial W}{\partial t}=C_{1} t^{K_{e}-1}
$$

where $\tau$ refers to the time coordinate and $t$ stands for the distance along the central streamline.

Integrating equation (1) and substituting the following boundary conditions,

$$
\begin{aligned}
& \mathrm{W}=\mathrm{W}_{3}, \text { at } \mathrm{t}=0, \text { and } \\
& \mathrm{W}=\mathrm{W}_{2} \text { at } \mathrm{t}=\mathrm{t}_{2},
\end{aligned}
$$

The solution can be written as,

$$
W^{2}=W_{3}^{2}+\left(W_{2}^{2}-W_{3}^{2}\right)\left(\frac{t}{t_{2}}\right)^{K_{e}}
$$

(ii) Pressure is constant over the blade channel in the direction normal to the mean relative streamline, indicating the hydrostatic pressure has negligible effect. Thus,

$$
\frac{\partial p}{\partial n}=0
$$

(iii) Relative flow angle at the wheel inlet $=90^{\circ}$.

Both the earlier conditions relate to the width of the channel and shape of the curve in meridian section. Another equation has to be formed which determine the shape of the curve in the circumferential direction. The variation of the relative velocity angle $\beta$ along the flow path follows the relation:

$$
\operatorname{cosec} \beta=\frac{\partial t}{\partial s}=\operatorname{cosec} \beta_{2}+C_{2}\left(1-\frac{s}{s_{2}}\right)^{K_{h}}
$$

where, $C_{2}=\operatorname{Cosec} \beta_{3}-\operatorname{Cosec} \beta_{2}$

(iv) Equal meridional velocity at the wheel inlet and at exit

$$
\text { The meridional velocity ratio: } k_{I}=\frac{C_{m 3}}{C_{m 2}}=1
$$

\subsection{Input and output variables}

The following tables give the list of input and output variables considered in the analysis. Consistent SI units have been used in all cases. 
A. Variable

Table 1. Input data for blade profile analysis of expansion turbine

\begin{tabular}{|l|l|l|}
\hline Variables & Notation & Units \\
\hline Free parameter & $k_{e}$ & None \\
\hline Free parameter & $k_{h}$ & None \\
\hline Characteristic angle & $\delta_{3}$ & radian \\
\hline Meridional streamlength & $s$ & $\mathrm{M}$ \\
\hline No of points for calculation & $\mathrm{n}$ points & None \\
\hline
\end{tabular}

B. Constant thermodynamic properties

\begin{tabular}{|l|l|l|}
\hline Constants & Notation & Units \\
\hline Outlet temperature & $T_{3}$ & $\mathrm{~K}$ \\
\hline Outlet pressure & $p_{3}$ & $\mathrm{~Pa}$ \\
\hline Outlet density & $\rho_{3}$ & $\mathrm{~kg} / \mathrm{m}^{3}$ \\
\hline Mass flow rate & $\dot{m}$ & $\mathrm{Kg} / \mathrm{s}$ \\
\hline
\end{tabular}

C. Constant fluid properties

\begin{tabular}{|l|l|l|}
\hline Property & Notation & Unit \\
\hline Specific heat ratio & $\gamma$ & None \\
\hline Polytropic Index & $m$ & None \\
\hline
\end{tabular}

D. Geometric inputs

\begin{tabular}{|l|l|l|l|}
\hline Component & Dimension & Notation & Unit \\
\hline \multirow{4}{*}{$\begin{array}{l}\text { Turbine } \\
\text { Wheel }\end{array}$} & Inlet radius & $r_{2}$ & $\mathrm{~m}$ \\
\cline { 2 - 4 } & Tip radius & $r_{t i p}$ & $\mathrm{~m}$ \\
\cline { 2 - 4 } & Hub radius & $r_{\text {hub }}$ & $\mathrm{m}$ \\
\cline { 2 - 4 } & No of blades & $Z_{t r}$ & None \\
\cline { 2 - 4 } & Blade thickness & $t_{t r}$ & $\mathrm{~m}$ \\
\cline { 2 - 4 } & Relative velocity angle & $\beta_{2}$ & Radian \\
\cline { 2 - 4 } & Exit Mean relative velocity angle & $\beta_{\text {mean }}$ & Radian \\
\hline
\end{tabular}

E. Constant design data

\begin{tabular}{|l|l|l|l|}
\hline Component & Constant & Notation & Unit \\
\hline \multirow{4}{*}{ Wheel } & Rotational speed & $\omega$ & $\mathrm{rad} / \mathrm{s}$ \\
\cline { 2 - 4 } & Exit absolute velocity & $C_{3}$ & $\mathrm{~m} / \mathrm{s}$ \\
\cline { 2 - 4 } & Exit circumferential velocity & $U_{3}$ & $\mathrm{~m} / \mathrm{s}$ \\
\cline { 2 - 4 } & Exit relative velocity & $W_{3}$ & $\mathrm{~m} / \mathrm{s}$ \\
\cline { 2 - 4 } & Exit sound velocity & $C_{s 3}$ & $\mathrm{~m} / \mathrm{s}$ \\
\hline
\end{tabular}


Table 2. Output variables in meanline analysis of expansion turbine performance

\begin{tabular}{|l|l|l|}
\hline Variables & Notation & Units \\
\hline Radial Co-ordinate along meridional streamlength & $r$ & $\mathrm{~m}$ \\
\hline Tangential Co-ordinate along meridional streamlength & $\theta$ & radian \\
\hline Axial Co-ordinate along meridional streamlength & $Z$ & $\mathrm{~m}$ \\
\hline Characteristic angle along meridional streamlength & $\delta$ & $\mathrm{radian}$ \\
\hline Relative velocity angle along meridional streamlength & $\beta$ & radian \\
\hline Absolute velocity along meridional streamlength & $C$ & $\mathrm{~m} / \mathrm{s}$ \\
\hline Relative velocity along meridional streamlength & $W$ & $\mathrm{~m} / \mathrm{s}$ \\
\hline Circumferential velocity along meridional streamlength & $U$ & $\mathrm{~m} / \mathrm{s}$ \\
\hline Pressure along meridional streamlength & $P$ & $\mathrm{bar}$ \\
\hline Temperature along meridional streamlength & $T$ & $\mathrm{~K}$ \\
\hline Density along meridional streamlength & $\rho$ & $\mathrm{Kg} / \mathrm{m}^{3}$ \\
\hline
\end{tabular}

\subsection{Governing equations}

To calculate the $\mathrm{r}, \theta$, $\mathrm{z}$ coordinate of the central streamline some input parameters like major dimensions of the flow conditions at wheel inlet and exit are required. The distance is along the meridional curve is taken as the independent variable. Integration proceeds from the exit end with the boundary conditions $\mathrm{s}=\mathrm{s}_{3}=0, \mathrm{r}=\mathrm{r}_{3}, \mathrm{z}=\mathrm{z}_{3}=0$ and $\delta=\delta_{\text {outlet }}$ till $\mathrm{s}=\mathrm{s}_{2}$. The solution process terminates when $\mathrm{r}=\mathrm{D} / 2$ and $\beta_{2}=90^{\circ}$.

Hasselgruber's formulation leads to three characteristic functions defined as follows.

$$
\mathrm{f}_{1}\left(\frac{\mathrm{s}}{\mathrm{s}_{2}}\right)=\sqrt{\left(\operatorname{cosec}\left(\beta_{\text {mean }}\right)\right)^{2}+\left\{\left(\operatorname{cosec}\left(\beta_{2}\right)\right)^{2}-\left(\operatorname{cosec}\left(\beta_{\text {mean }}\right)\right)^{2}\right\} \times \mathrm{A}}
$$

where,

$$
\begin{aligned}
& \mathrm{A}=\left[\frac{\frac{\mathrm{s}}{\mathrm{s}_{2}} \times\left(\mathrm{k}_{\mathrm{h}}+1\right) \times \operatorname{cosec}\left(\beta_{2}\right)+\left(\operatorname{cosec}\left(\beta_{\text {mean }}\right)-\operatorname{cosec}\left(\beta_{2}\right)\right) \times\left\{1-\left(1-\frac{\mathrm{s}}{\mathrm{s}_{2}}\right)^{k_{\mathrm{h}}+1}\right\}}{\mathrm{k}_{\mathrm{h}} \times \operatorname{cosec}\left(\beta_{2}\right)+\operatorname{cosec}\left(\beta_{\text {mean }}\right)}\right]^{k_{e}} \\
& \mathrm{f}_{2}\left(\frac{\mathrm{s}}{\mathrm{s}_{2}}\right)=\frac{1}{\operatorname{cosec}\left(\beta_{2}\right)+\left\{\operatorname{cosec}\left(\beta_{\text {mean }}\right)-\operatorname{cosec}\left(\beta_{2}\right)\right\} \times\left(1-\frac{\mathrm{s}}{\mathrm{s}_{2}}\right)^{k_{h}}} \\
& \mathrm{f}_{3}\left(\frac{\mathrm{s}}{\mathrm{s}_{2}}\right)=\mathrm{f}_{1}\left(\frac{\mathrm{s}}{\mathrm{s}_{2}}\right) \times \sqrt{1-\mathrm{f}_{2}^{2}\left(\frac{\mathrm{s}}{\mathrm{s}_{2}}\right)}
\end{aligned}
$$

The function $\mathrm{f}_{1}$ depicts the variation of the relative acceleration of the fluid from the wheel inlet to exit. The function $\mathrm{f}_{2}$ gives the relative flow angle along the flow path while function $f_{3}$ is a combination of $f_{1} \& f_{2}$.

The radius of curvature of meridional streamline path is expressed in terms of the three characteristic functions

$$
\mathrm{R}_{\mathrm{m}}=\left[\frac{\mathrm{f}_{1}\left(\frac{\mathrm{s}}{\mathrm{s}_{2}}\right) \times \mathrm{f}_{2}\left(\frac{\mathrm{s}}{\mathrm{s}_{2}}\right)}{\frac{\mathrm{r}}{\mathrm{r}_{\text {mean }} \times \tan \left(\beta_{\text {mean }}\right)}-\mathrm{f}_{3}\left(\frac{\mathrm{s}}{\mathrm{s}_{2}}\right)}\right]^{2} \times \frac{\mathrm{r}}{\cos (\delta)}
$$

where, $\quad r_{\text {mean }}=0.5\left(r_{\text {tip }}+r_{\text {hub }}\right)$

The angle between meridional velocity component and axial coordinate is derived to be: 


$$
\delta=\int_{0}^{s}\left(\frac{1}{R_{m}}\right) d s
$$

The coordinate $(\mathrm{r}, \theta, \mathrm{z})$ of the central streamline are calculated by using the following equations:

$$
\begin{aligned}
& r=\int_{0}^{s}(\sin \delta) d s \\
& \theta=\int_{0}^{\mathrm{s}}\left(\frac{\sqrt{1-\mathrm{f}_{2}^{2}\left(\frac{\mathrm{s}}{\mathrm{s}_{2}}\right)}}{\mathrm{r} \times \mathrm{f}_{2}\left(\frac{\mathrm{s}}{\mathrm{s}_{2}}\right)}\right) \mathrm{ds} \\
& z=\int_{0}^{s}(\cos \delta) d s
\end{aligned}
$$

Middle stream-surface is created by joining the points on the hub streamline to the corresponding points on the tip streamline. The coordinates of hub and tip streamlines are calculated by using channel depth and the angle between meridional velocity component and axial coordinate.

To represent the ratio of meridian to circumferential velocity, a characteristics factor is defined as:

$$
\lambda_{m}=\frac{C_{m 3}}{U_{3}}=\tan \beta_{3}
$$

The velocities at different points of meridional streamline are

$$
\begin{aligned}
& W_{m}=C_{m 3} \times f_{1}\left(\frac{s}{s_{2}}\right) \\
& \beta_{m}=\sin ^{-1} f_{2}\left(\frac{s}{s_{2}}\right) \\
& U_{m}=\frac{U_{3} \times r}{r_{\text {mean }}} \\
& C_{u}=U-W \cos \beta \\
& C_{m}=C_{m 3} \times f_{1}\left(\frac{s}{s_{2}}\right) \times f_{2}\left(\frac{s}{s_{2}}\right) \\
& C=\sqrt{\left(C_{m}{ }^{2}+C_{u}{ }^{2}\right)}
\end{aligned}
$$

The density along the fluid flow path is

$$
\rho=\rho_{3} \times\left(1+M_{3}^{2} \times \frac{(m-1) \gamma}{2 m} \times \frac{U^{2}-W^{2}-U_{3}^{2}+W_{3}^{2}}{C_{m 3}{ }^{2}}\right)^{\frac{1}{m-1}}
$$

The channel width and channel depth at each point is determined by using these equations respectively as,

$$
w_{t r}=\frac{2 \pi r \sin \beta-Z_{t r} t_{t r}}{Z_{t r}}
$$




$$
\Delta b=\frac{\dot{m} \operatorname{tr} \sin \beta}{Z_{t r} w_{t r} \rho C_{m}}
$$

The $\mathrm{r}, \theta$, $\mathrm{z}$ coordinate of the hub and tip streamlines are calculated by using the following equations:

$$
\left.\begin{array}{l}
\mathrm{r}_{\text {hub }}=\mathrm{r}_{\text {mean }}-\frac{\Delta \mathrm{b}}{2} \times \cos (\delta) \\
\mathrm{r}_{\text {tip }}=\mathrm{r}_{\text {mean }}+\frac{\Delta \mathrm{b}}{2} \times \cos (\delta) \\
\mathrm{z}_{\text {hub }}=\mathrm{z}_{\text {mean }}+\frac{\Delta \mathrm{b}}{2} \times \sin (\delta) \\
\mathrm{z}_{\text {tip }}=\mathrm{z}_{\text {mean }}-\frac{\Delta \mathrm{b}}{2} \times \sin (\delta) \\
\theta_{\text {tip }}=\theta_{\text {hub }}=\theta_{\text {mean }}
\end{array}\right\}
$$

The surface so generated is considered as the mean surface within a blade. The suction and pressure surfaces of two adjacent channels are computed by translating the mean surface in the $+v e$ and -ve $\theta$ directions through half the blade thickness. The suction side and pressure side surfaces ( $\mathrm{r}, \theta$ and $\mathrm{z}$ coordinates of streamlines) are obtained through the following equations:

$$
\begin{aligned}
& \mathrm{r}_{\text {pressure }}=\mathrm{r}_{\text {suction }}=\mathrm{r}_{\text {mean }} \\
& \mathrm{z}_{\text {pressure }}=\mathrm{z}_{\text {suction }}=\mathrm{z}_{\text {mean }} \\
& \theta_{\text {pressure }}=\theta_{\text {mean }}+\frac{t_{\text {tr }}}{2 * r_{\text {mean }}} * \cos \beta \\
& \theta_{\text {suction }}=\theta_{\text {mean }}-\frac{t_{\text {tr }}}{2 * r_{\text {mean }}} * \cos \beta
\end{aligned}
$$

The average velocity can be split into two parts: one, due to the curvature of the blades and other, due to the rotation of the blades - the so-called channel-vortex. For turbine wheel as the curvature is backward ( $R_{b}$ is positive), the effects are opposite.

The blade angle $\beta$ along the hub and tip streamlines are calculated by using the following equations:

$$
\begin{aligned}
& \beta_{h u b}=\tan ^{-1}\left[\frac{r}{r_{h u b}} \times \tan \beta\right] \\
& \beta_{t i p}=\tan ^{-1}\left[\frac{r}{r_{t i p}} \times \tan \beta\right]
\end{aligned}
$$

The velocities of fluid at the suction and pressure side are determined from the following expression.

$$
\begin{aligned}
w_{p s, s s}= & w \cdot\left(1 \pm \frac{L}{2 \cdot R_{b}}\right) \mp \omega \cdot L \cdot \sin \delta \\
\text { where, } \quad R_{b} & =\left(-\frac{s_{2}}{K_{h}}\right) \times \frac{\operatorname{cosec} c^{2} \beta}{C\left(1-\frac{s}{s_{2}}\right)^{K_{h}-1}} \times \cot \beta \\
C & =\frac{\left(\sqrt{1+\lambda_{1}^{2}}-\lambda_{1}\right)}{\lambda_{1}} \\
\lambda_{1} & =\tan \beta_{3}
\end{aligned}
$$




\subsection{Outline of computer programme}

- Inputs: major dimensions and the flow conditions at the inlet and exit of the wheel

- Determination of the middle streamline

- Determination of the hub and tip streamlines

- Determination of the suction and pressure side geometry of the blade

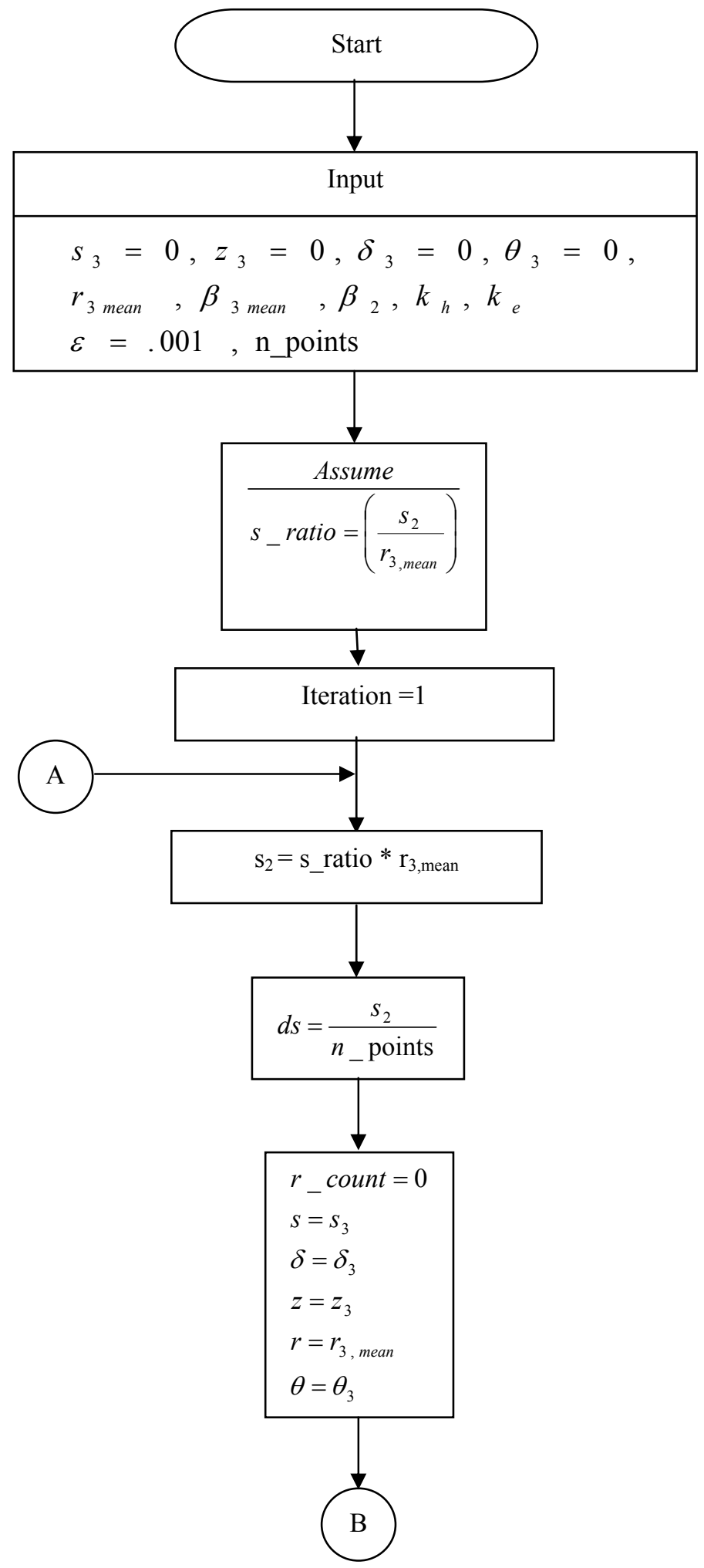




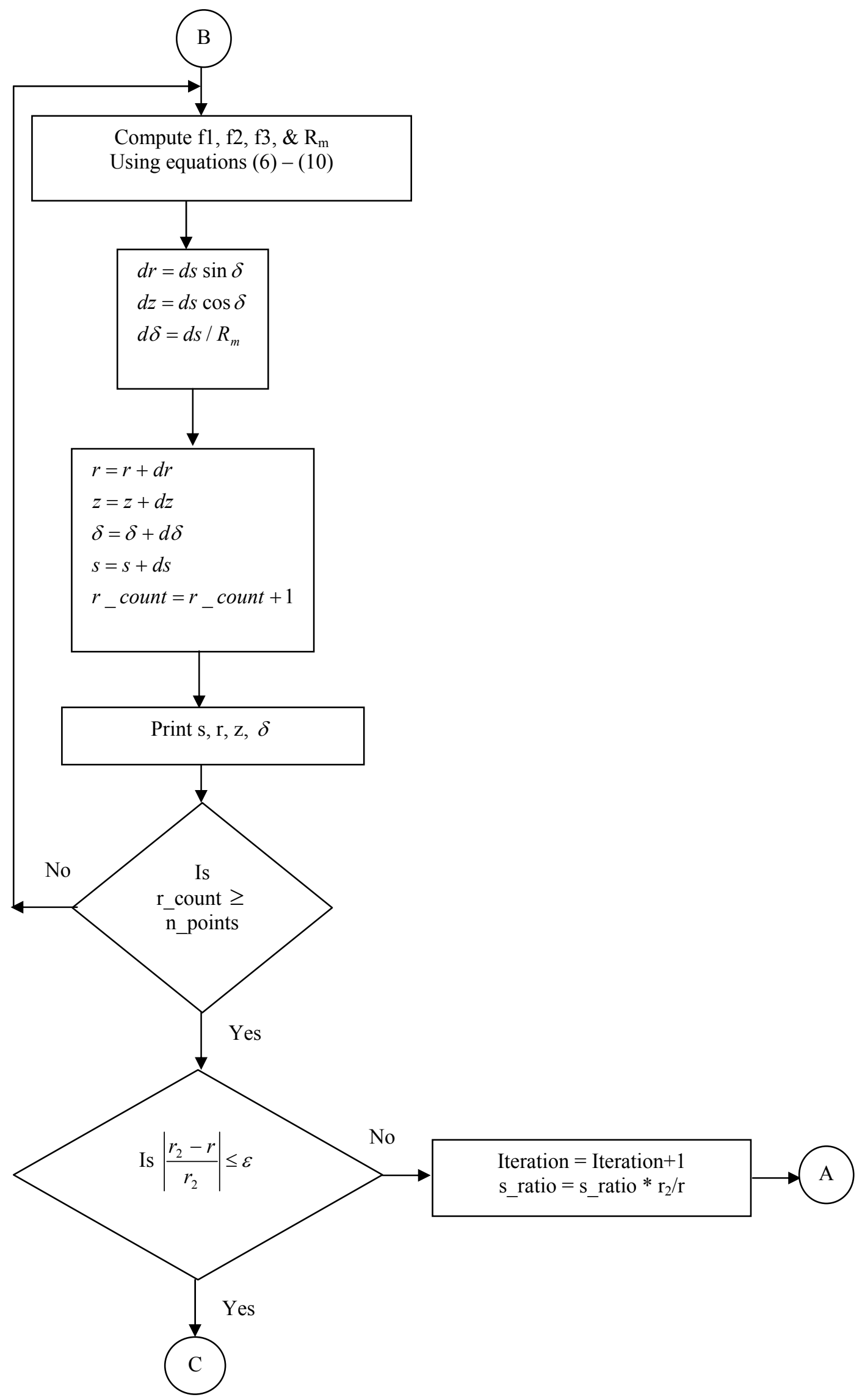




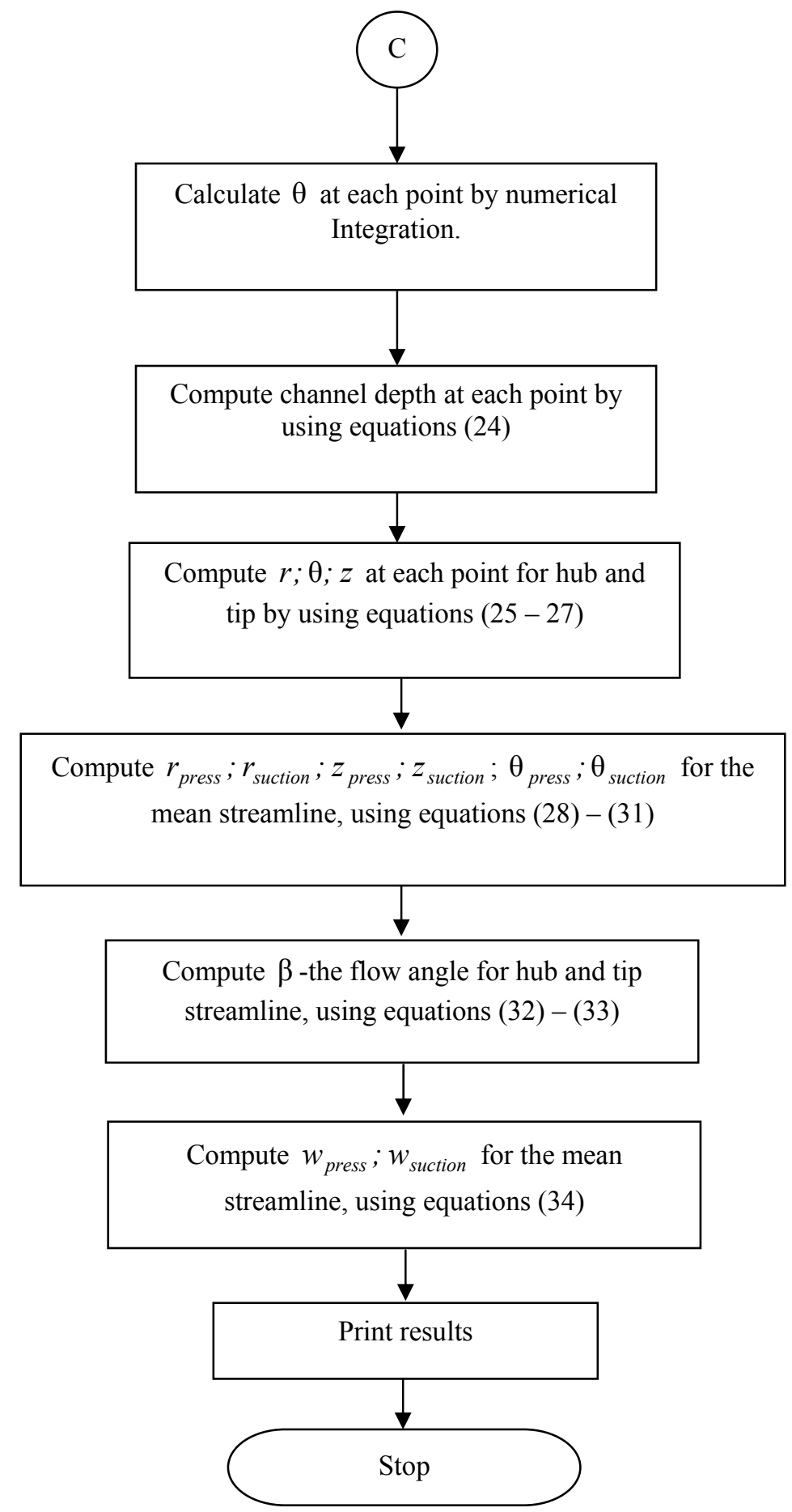

Figure 3. Flow chart of the computer program for calculation of blade profile using Hasselgruber's method

\section{Results and discussion}

There are two free parameters for calculating the flow path contour $k_{h}$ and $k_{e}$. The parameter $k_{h}$ controls the change in flow path angle whereas the term $k_{e}$ is the acceleration exponent which controls the relative acceleration. Effect of these two parameters on blade profile discussed below. 


\section{Variation of $k_{h}$}

As $k_{h}$ may vary from 1 to 20 , the greater the $k_{h}$, greater is the curvature of the blade at the wheel exit, shown in Figure 4 . More compact is the construction, the shorter is the path of the stream. So, the distribution of the velocity at the wheel exit is nonuniform and this means a strong friction. Again the probability of dumb-bell shaped blade is more in case of large $k_{h}$ value. The net effect is the worsening of the efficiency. The axial co-ordinate does not change too much with changing of $k_{h}$ as shown in Figure 7. But the angular co-ordinate of blade will be more in case of small $k_{h}$ value as shown in Figure 10, which is not permitted. The characteristic angle ( $\delta_{3}$, Figure 13) is more for large $k_{h}$ value i.e. to achieve the value $\delta_{2}$ as $90^{\circ}$, it is required to choose more $k_{h}$. To calculate the flow angle from exit to inlet it has been seen from Figure 16 that, for small $k_{h}$, it is increasing at a particular slope initially but at close to the inlet, it changes abruptly. Again for the large value of $k_{h}$ the flow angle increasing to the particular value and then it remains unchage.

Variation of $k_{e}$

The radial co-ordinate, axial co-ordinate and the angular co-ordinate do not change significantly with changing of $k_{e}$ as shown in Figure 5, Figure 8 and Figure 11. As shown in Figure 14 the characteristic angle $\delta_{2}$ is more in case of small $k_{e}$ and it decreases for large $k_{e}$. The flow angle is totally independent of $k_{e}$ is shown in Figure 17. For $k_{e}>1$ the relative acceleration i.e. the rate of change of relative velocity along the meridian streamlength from inlet to exit is more and effect is less for $k_{e}<1$.

\section{Variation of $\delta_{3}$}

By allowing a smaller $\delta_{3}$ at the exit of the wheel, the curvature of the blade is smaller at the wheel exit as shown in Figure 6 and the chances of dumb-bell shaped can be eliminated. The axial co-ordinate and the angular co-ordinate are also less in case of some value of $\delta_{3}$ as shown in Figure 9 and Figure 12. But the characteristics angle at the inlet of the turbine wheel will be more for more $\delta_{3}$ as shown in Figure 15. The flow angle is almost independent on the initial value of characteristic angle as shown in Figure 18.

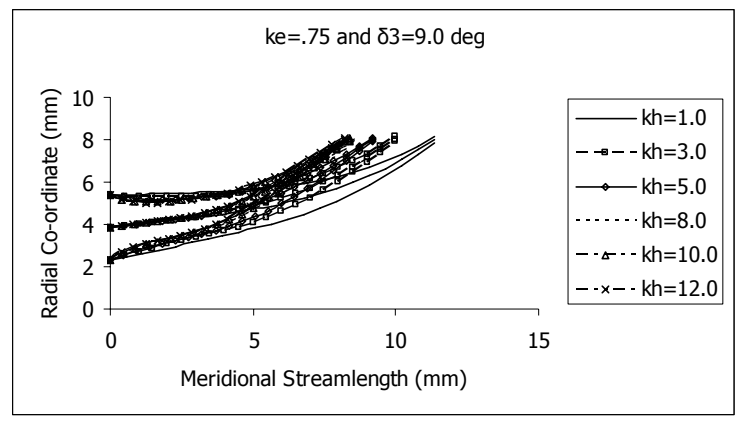

Figure 4. Variation of radial co-ordinate of turbine wheel with the variation of $k_{h}$

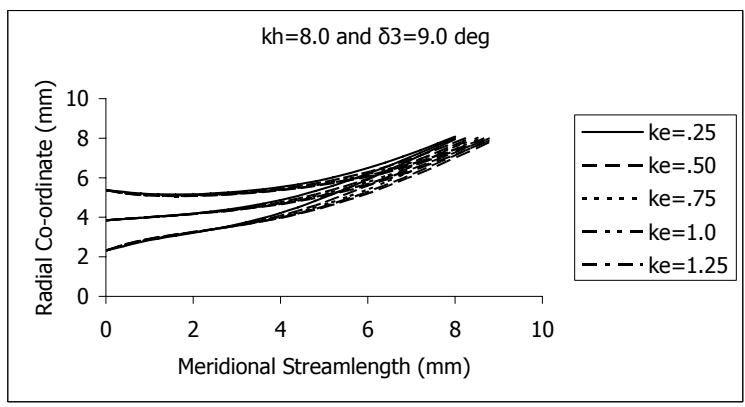

Figure 5. Variation of radial co-ordinate of turbine wheel with the variation of $k_{e}$ 


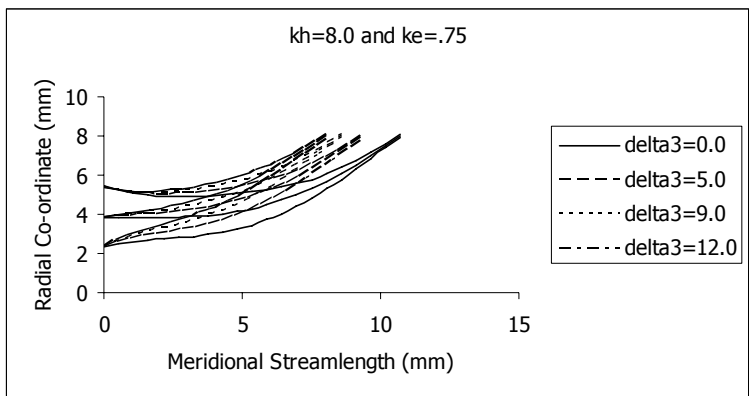

Figure 6. Variation of radial co-ordinate of turbine wheel with the variation of $\delta_{3}$

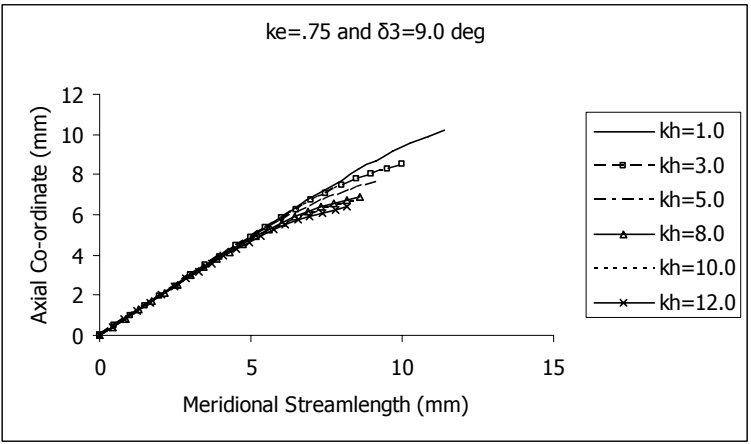

Figure 7. Variation of axial co-ordinate of turbine wheel with the variation of $k_{h}$

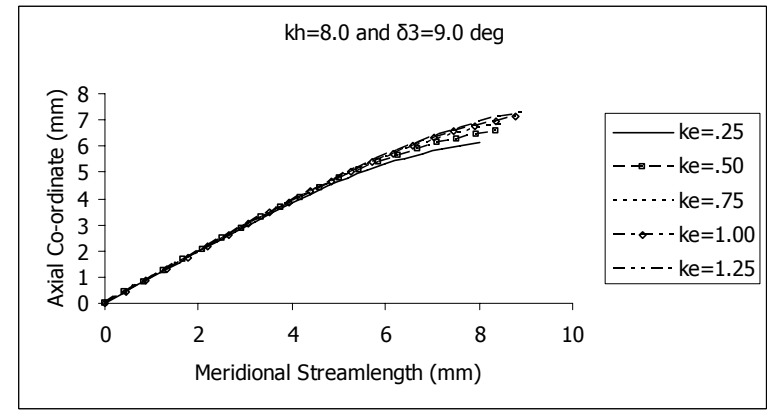

Figure 8. Variation of axial co-ordinate of turbine wheel with the variation of $k_{e}$

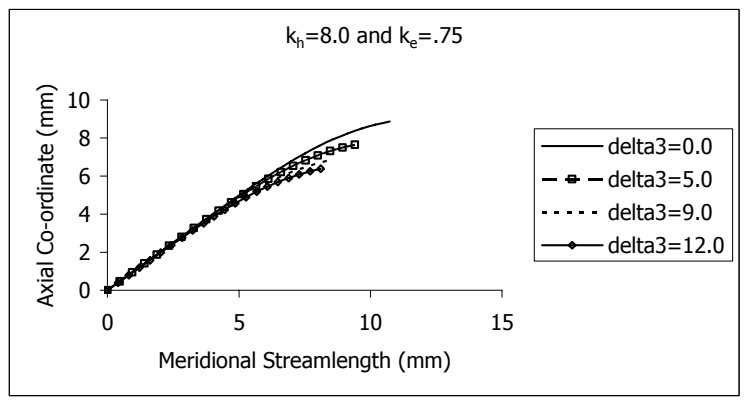

Figure 9. Variation of axial co-ordinate of turbine wheel with the variation of $\delta_{3}$ 


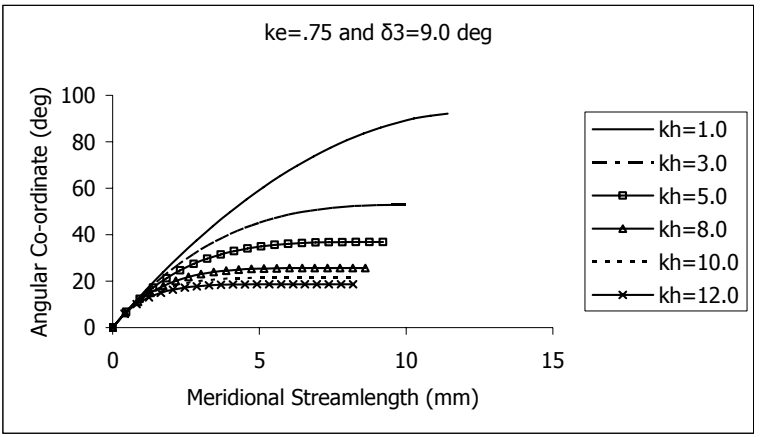

Figure 10. Variation of angular co-ordinate of turbine wheel with the variation of $k_{h}$

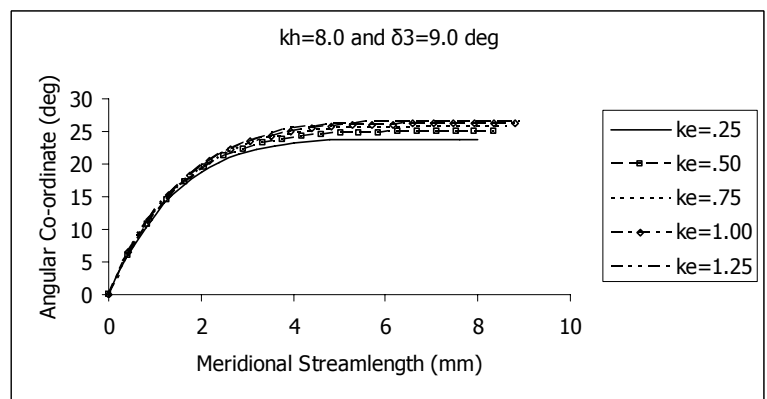

Figure 11. Variation of angular co-ordinate of turbine wheel with the variation of $k_{e}$

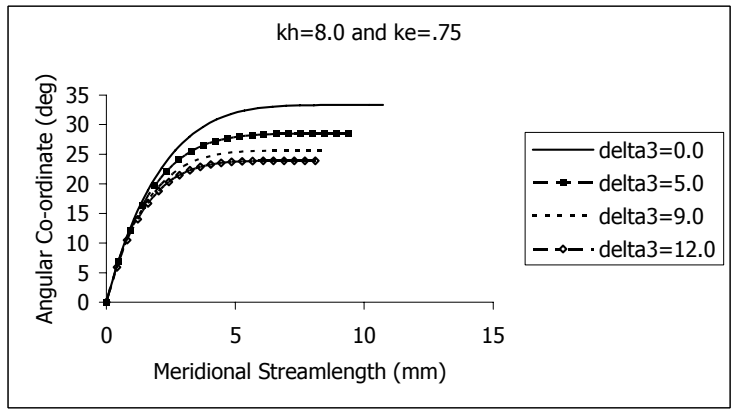

Figure 12. Variation of angular co-ordinate of turbine wheel with the variation of $\delta_{3}$

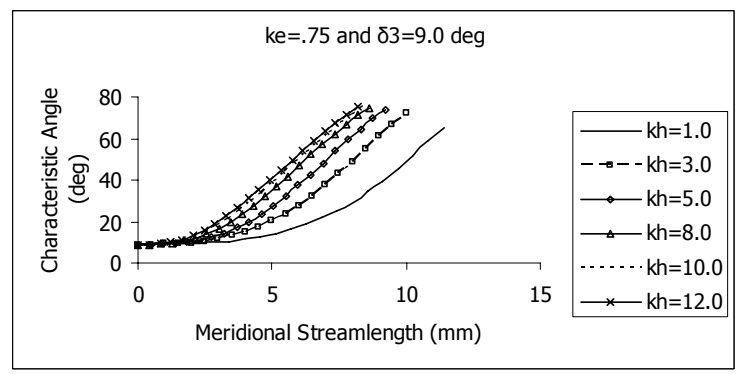

Figure 13. Variation of characteristic angle in the turbine wheel with the variation of $k_{h}$ 


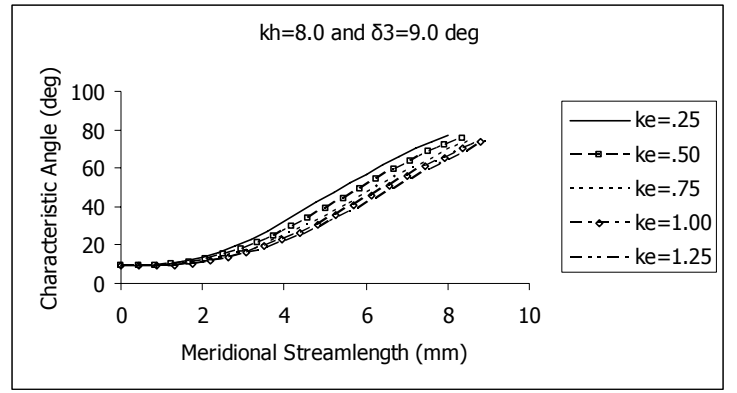

Figure 14. Variation of characteristic angle in the turbine wheel with the variation of $k_{e}$

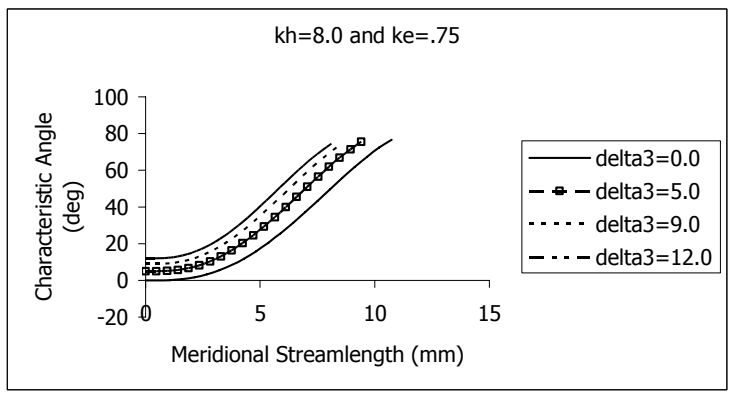

Figure 15. Variation of characteristic angle in the turbine wheel with the variation of $\delta_{3}$

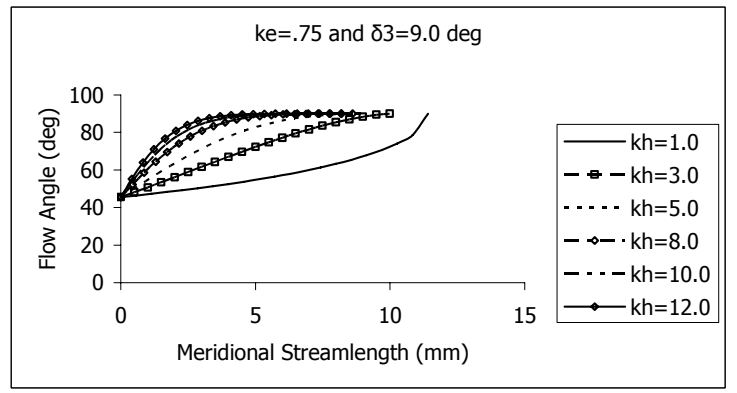

Figure 16. Variation of flow angle in the turbine wheel with the variation of $k_{h}$

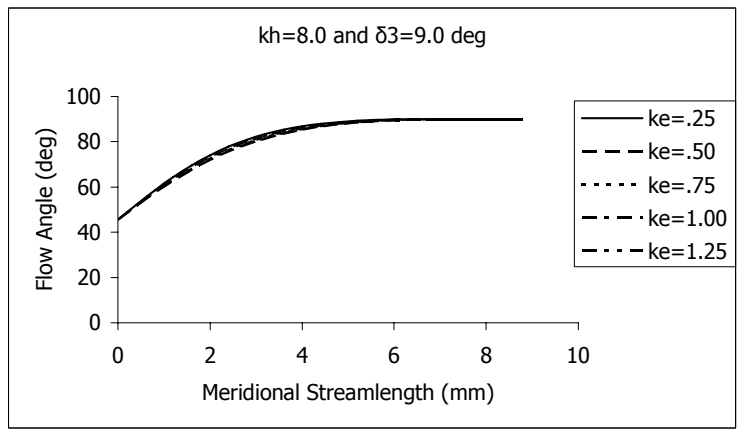

Figure 17. Variation of flow angle in the turbine wheel with the variation of $k_{e}$ 


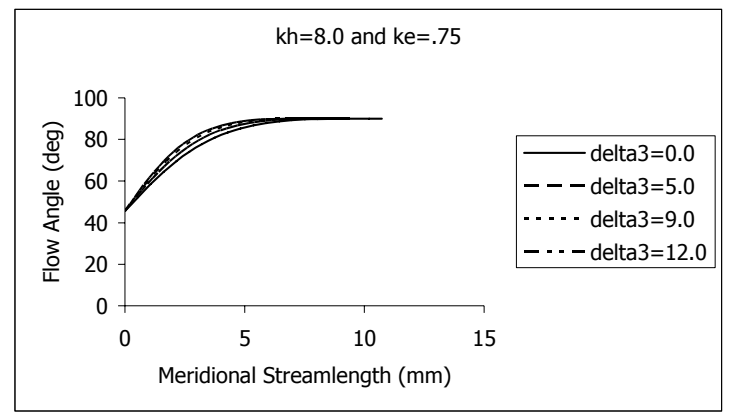

Figure 18. Variation of flow angle in the turbine wheel with the variation of $\delta_{3}$

\section{Conclusions}

A numerical model of the cryogenic turbine wheel blade geometry has been developed covering meridional, suction, pressure, hub and tip streamline to study the influence of design and operating parameters on the shape of the blade. In addition to its use in the definition of impeller geometry, the method can be used to define the complete flow passages in a turbine. By using this method the designer is able to get a better feel for the geometry during the design process, he spends less time on mundane data preparation, and he has more freedom to make full use of this creativity to design a better machine. Analysis of result reveals that the optimum value exists to avoid the dumb-bell shaped blade and to have an optimum path length. One of the optimum value of $k_{h}=5.0, k_{e}=0.75$ leads to exit radial component $\delta_{3}$ as $9^{\circ}$.

\section{Nomenclature}

b channel width

C absolute velocity

$\mathrm{D}_{2} \quad$ turbine wheel diameter

$\mathrm{D}_{\text {tip }} \quad$ eye tip diameter

$\mathrm{D}_{\text {hub }} \quad$ eye hub diameter

$\mathrm{d}_{\mathrm{s}} \quad$ specific diameter

h enthalpy

$\Delta \mathrm{h}_{\text {in-3s }} \quad$ adiabatic enthalpy drop across turbine wheel

$\mathrm{K}_{\mathrm{e}} \quad$ free parameter

$\mathrm{K}_{\mathrm{h}} \quad$ free parameter

m mass flow rate

M mach number

$\mathrm{N}$ number of revolution

$\mathrm{n}_{\mathrm{s}} \quad$ specific speed

$\mathrm{P} \quad$ power produced

$\mathrm{p} \quad$ pressure

Q volumetric flow rate

$\mathrm{R}_{\mathrm{m}} \quad$ radius of curvature of meridional streamline

$\mathrm{r} \quad$ radius

$\mathrm{S}$ direction and arc length of a meridional streamline

s entropy

$t_{b} \quad$ blade thickness

$\mathrm{T}$ temperature

U circumferential velocity

W relative velocity

$\mathrm{Z} \quad$ number of blades

$\mathrm{r}, \theta, \mathrm{z}$ cylindrical coordinate fixed to rotor

\section{Greek Symbols}

$\eta \quad$ efficiency

$\omega \quad$ rotational speed 
$\varepsilon \quad$ ratio of tip diameter to turbine wheel diameter

$\lambda \quad$ ratio of hub diameter to tip diameter

$\beta \quad$ relative velocity angle

$\tau \quad$ time coordinate

$\delta \quad$ angle between meridional velocity component and axial coordinate

$\rho \quad$ density of fluid

$\begin{array}{ll}\text { Subscripts } \\ \text { in } & \text { inlet to nozzle } \\ 1 & \text { exit to nozzle } \\ 2 & \text { inlet to turbine wheel } \\ 3 & \text { exit to turbine wheel } \\ \text { ex } & \text { exit from diffuser } \\ \text { hub } & \text { hub of turbine wheel at exit } \\ \text { tip } & \text { tip of turbine wheel at exit } \\ \mathrm{m} & \text { meridional } \\ \mathrm{u} & \text { circumferential } \\ \mathrm{tr} & \text { turbine } \\ \mathrm{s} & \text { isentropic condition } \\ 0 & \text { stagnation condition }\end{array}$

\section{Acknowledgements}

The authors are grateful to the Board of Research in Nuclear Sciences (Department Atomic Energy) for sponsoring this work. The technical inputs from Mr. Trilok Singh, Head Cryo Technology Division, BARC and his group are also gratefully acknowledged. The authors are thankful to the Mechanical Engineering Department, NIT, Rourkela, for providing all testing facilities and guidance.

\section{References}

Awatef A. Hamed, Widen Tabakoff, Richard B. Rivir, Kaushik Das, Puneet Arora, 2005, Turbine Blade Surface Deterioration by Erosion, ASME, Journal of Turbomachinery, Vol. 27, pp. 445-452

Balje, O. E. 1970, Loss and flow path studies on centrifugal compressors, Part-II, Trans ASME J Eng Power, pp. $275-299$.

Balje, O.E. 1981. Turbomachines: A guide to design, selection and theory. John Wiley and Sons, New York.

Bruce, G. J. 1998, Computer-aided turbomachinery design system, I Mech E Conference on Turbochargers I Mech E Publications (Paper No. C554/026/98. pp. 3-22).

Casey, M. V. A 1983. Computational geometry for the blades and internal flow channels of centrifugal compressors, Trans ASME J Eng Power, V105, pp. 288-295

Denton, J. D. 1996, The turboexpander-a design, make and test student project ASME-96-GT- 191.

Dixon, S. L. 1978, Fluid mechanics and thermodynamics of turbomachinery ( $3^{\text {rd }}$ ed) Elsevier, USA.

Hasselgruber,H. 1958, Stromungsgerechte gestaltung der laufrader von radialkompressoren mit axialem laufradeintrict Konstruction, Vol. 10, No. 1, pp. 22 (in German).

Ito E., Sakamoto Y., Tsukagoshi K., Muyama A., Masada J., 2009, Advanced three-dimensional aerodynamic design for next generation gas turbines, Mitsubishi Heavy Industries Technical Review, Vol. 46 No. 1, pp. 9-11.

Kun, L .C. and Sentz, R. N. 1985, High efficiency expansion turbines in air separation and liquefaction plants, International Conference on Production and Purification of Coal Gas \& Separation of Air, Beijing, China, pp.1-21

RohliK, H.E. 1968, Analytical determination of radial inflow turbine geometry for maximum efficiency NASA TN D-4384.

Strinning, P. 1995, A computerised trial and error technique for impeller design using computers in the design and selection of fluid machinery, I Mech E, No. 3, pp. 19-27.

Thakker A. and Abdulhadi R., 2007, Effect of blade profile on the performance of wells turbine under unidirectional sinusoidal and real sea flow conditions, International Journal of Rotating Machinery, pp. 1-8.

Wallace, F.J., Whitfield, A. and Atkey, R. 1975, A computer-aided design for radial and mixed flow compressor, Computer Aided Design, pp. 163-170.

Whitfield, A. 1978, Computer aided design and testing of radial and centrifugal impellers with straight and backward swept blades, Proc Inst Mech Eng, pp. 89-100. 


\section{Biographical notes}

Dr. Subrata Kumar Ghosh is Senior Lecturer in the Department of Mechanical Engineering \& Mining Machinery Engineering at ISMU, University, Dhanbad, India. Before joining at ISMU He was Lecturer at BIT, Mesra. His research has focused on Thermal Engineering. He is the author and coauthor of over 8 articles, which have appeared in journals such as Applied Thermal Engineering, Indian Journal of Cryogenics, and several seminars.

Prof. Ranjit Kr. Sahoo is Professor and Head of Mechanical Engineering Department in the National Institute of Technology, Rourkela, India. His research has focused on Heat Transfer, Cryogenic Engineering in Space Applications etc. He is the author of over 20 articles, which have appeared in reputed International Journals.

Prof. Sunil Kr. Sarangi is Director of the National Institute of Technology, Rourkela, India. He is also Professor at the Cryogenic Centre of Indian Institute of Technology, Kharagpur, India. His research has focused on Cryogenic Engineering in Space Applications, as well as Heat Exchanger, Radiation etc. He is the author of over 50 articles, which have appeared in reputed International Journals.

Received July 2009

Accepted September 2009

Final acceptance in revised form September 2009 